\title{
CONTROLLABILITY FOR SEMILINEAR FUNCTIONAL INTEGRODIFFERENTIAL EQUATIONS
}

\author{
Jin-Mun JeOng And Han-Geul Kim
}

\begin{abstract}
This paper deals with the regularity properties for a class of semilinear integrodifferential functional differential equations. It is shown the relation between the reachable set of the semilinear system and that of its corresponding linear system. We also show that the Lipschitz continuity and the uniform boundedness of the nonlinear term can be considerably weakened. Finally, a simple example to which our main result can be applied is given.
\end{abstract}

\section{Introduction}

Let $H$ and $V$ be two complex Hilbert spaces such that $V$ is a dense subspace of $H$. Identifying the antidual of $H$ with $H$ we may consider $V \subset H \subset V^{*}$.

In this paper we deal with the approximate controllability for the semilinear equation in $H$ as follows.

$$
\begin{cases}\frac{d}{d t} x(t) & =A x(t)+\int_{0}^{t} k(t-s) g(s, x(s), u(s)) d s+B u(t), \\ x(0) & =x_{0} .\end{cases}
$$

Here, the nonlinear part is given by

$$
f(t, x, u)=\int_{0}^{t} k(t-s) g(s, x(s), u(s)) d s,
$$

where $k$ belongs to $L^{2}(0, T)$ and $g:[0, T] \times V \times U \rightarrow H$ is a nonlinear mapping satisfying Lipschitz continuous, that is, there exist positive constants $L_{1}, L_{2}$ such that

$$
|g(t, x, u)-g(t, \hat{x}, \hat{u})| \leq L_{1}|| x-\hat{x}\left\|+L_{2}|| u-\hat{u}\right\|_{U} .
$$

In (SE), the principal operator $A$ generates an analytic semigroup $S(t)$ on $H$ and $B$ is a bounded linear operator from some Hilbert space $U$ to $H$. Let $x(T ; f, u)$ be a solution of (SE) associated with the nonlinear term $f$ and the

Received May 20, 2008.

2000 Mathematics Subject Classification. Primary 35B37, 35F25.

Key words and phrases. semilinear control systems, existence of solutions, regularity, approximate controllability.

This work was supported by the Korea Research Foundation Grant funded by the Korean Government (MOEHRD, Basic Research Promotion Fund) (KRF-2007-313-c00052)). 
control $u$ at the time $T$. We say that the system (SE) is approximate controllable on $[0, T]$ if for every desired final state $x_{1}$ and $\epsilon>0$ there exists a control function $u \in L^{2}(0, T ; U)$ such that $\left\|x(T ; f, u)-x_{1}\right\|_{H}<\epsilon$. Dauer and Mahmudov [2] dealt with the approximate controllability of a semilinear control system as a particular case of sufficient conditions for approximate solvability of semilinear equations by assuming $S(t)$ is compact operator for each $t>0, f$ is continuous and uniformly bounded and

(1) the corresponding linear system (SE) when $f \equiv 0$ is approximately controllable.

As for the some considerations on the trajectory set of (SE) and that of its corresponding linear system (in case $f \equiv 0$ ) as matters connected with (1), we refer to $[8,9]$ and references therein.

Sukavanam and Nutan Kumar Tomar [5] studied the approximate controllability for the following general retarded initial value problem:

$$
\left\{\begin{array}{l}
\frac{d x(t)}{d t}=A x(t)+B x(t)+f\left(t, x_{t}, u(t)\right), \quad 0<t \leq T, \\
x_{0}(0)=\phi(\theta), \quad-h \leq \theta \leq 0
\end{array}\right.
$$

in $C([-h, 0] ; V)$ by assuming $(1)$ and

(2) there exists a constant $\beta>0$ such that $\|B v\| \geq \beta\|v\|$ for all $v \in$ $L^{2}(0, T ; U)$ and $L_{1}<\beta$, where $L_{1}$ is the Lipschitz constant of $f$.

In this paper, we will deal with the control problems of (SE) on

$$
L^{2}(0, T ; V) \cap W^{1,2}\left(0, T ; V^{*}\right) \subset C([0, T] ; H) .
$$

So, we no longer require the compact property of semigroup, and the uniform boundedness and the inequality condition for Lipschitz continuity of $f$, but instead we need the regularity and a variation of solutions of the given equations in $L^{2}(0, T ; V)$. In Section 2, we will study wellposedness and regularity properties for a class of the semilinear control system. In Section 3, it is shown the relation between the reachable set of the semilinear system and that of its corresponding linear system. We also show that the Lipschitz continuity and the uniform boundedness of the nonlinear term assumed by $[5,8]$ can be considerably weakened. In last section, a simple example to which our main result can be applied is given.

\section{Semilinear functional equations}

Let $H$ and $V$ be complex Hilbert spaces such that $V \subset H \subset V^{*}$ by identifying the antidual of $H$ with $H$. Therefore, for the brevity, we may regard that $\|u\|_{*} \leq|u| \leq\|u\|$ for all $u \in V$, where the notations $|\cdot|,\|\cdot\|$ and $\|\cdot\|_{*}$ denote the norms of $H, V$ and $V^{*}$, respectively as usual. Let $a(u, v)$ be a bounded sesquilinear form defined in $V \times V$ satisfying Gårding's inequality

$$
\operatorname{Re} a(u, u) \geq c_{0}\|u\|^{2}-c_{1}|u|^{2}, \quad c_{0}>0, \quad c_{1} \geq 0 .
$$


Let $A$ be the operator associated with this sesquilinear form:

$$
(A u, v)=-a(u, v), \quad u, v \in V .
$$

Then $A$ is a bounded linear operator from $V$ to $V^{*}$. The realization of $A$ in $H$ which is the restriction of $A$ to

$$
D(A)=\{u \in V: A u \in H\}
$$

is also denoted by $A$. Therefore, in terms of the intermediate theory we can see that

$$
\left(V, V^{*}\right)_{1 / 2,2}=H,
$$

where $\left(V, V^{*}\right)_{\frac{1}{2}, 2}$ denotes the real interpolation space between $V$ and $V^{*}$ (see [7]). Moreover, for each $T>0$, by using interpolation theory we have

$$
L^{2}(0, T ; V) \cap W^{1,2}\left(0, T ; V^{*}\right) \subset C([0, T] ; H) .
$$

From the following inequalities

$$
\begin{aligned}
c_{0}|| u \|^{2} & \leq \operatorname{Re} a(u, u)+c_{1}|u|^{2} \leq C|A u||u|+c_{1}|u|^{2} \\
& \leq\left(C|A u|+c_{1}|u|\right)|u| \leq C|| u \|_{D(A)}|u|,
\end{aligned}
$$

it follows that there exists a constant $C_{0}>0$ such that

$$
\|u\| \leq C_{0}\|u\|_{D(A)}^{1 / 2}|u|^{1 / 2} .
$$

It is known that $A$ generates an analytic semigroup $S(t)$ in both $H$ and $V^{*}$. For the sake of simplicity we assume that $c_{1}=0$ and hence the closed half plane $\{\lambda: \operatorname{Re} \lambda \geq 0\}$ is contained in the resolvent set of $A$.

The following lemma is from Lemma 3.6.2 of [6].

Lemma 2.1. There exists a constant $M>0$ such that the following inequalities hold for all $t>0$ and every $x \in H$ or $V^{*}$ :

$$
\begin{aligned}
& |S(t) x| \leq M|x|, \\
& || S(t) x\left\|_{*} \leq M\right\| x \|_{*}, \\
& |S(t) x| \leq M t^{-1 / 2}\|x\|_{*}, \\
& || S(t) x \| \leq M t^{-1 / 2}|x| .
\end{aligned}
$$

The following initial value problem for the abstract linear parabolic equation

$$
\left\{\begin{aligned}
\frac{d x(t)}{d t} & =A x(t)+k(t), \quad 0<t \leq T, \\
x(0) & =x_{0} .
\end{aligned}\right.
$$

By virtue of Theorem 3.3 of [1] (or Theorem 3.1 of [3]), we have the following result on the corresponding linear equation of (LE).

Proposition 2.2. Suppose that the assumptions for the principal operator $A$ stated above are satisfied. Then the following properties hold: 
1) Let $F=(D(A), H)_{1 / 2,2}$, where $(D(A), H)_{1 / 2,2}$ is the real interpolation space between $D(A)$ and $H$ (see [7, Section 1.3.3]). For $x_{0} \in F$ and $k \in$ $L^{2}(0, T ; H), T>0$, there exists a unique solution $x$ of $(\mathrm{LE})$ belonging to

$$
L^{2}(0, T ; D(A)) \cap W^{1,2}(0, T ; H) \subset C([0, T] ; F)
$$

and satisfying

$$
\|x\|_{L^{2}(0, T ; D(A)) \cap W^{1,2}(0, T ; H)} \leq C_{1}\left(\left\|x_{0}\right\|_{F}+\|k\|_{L^{2}(0, T ; H)}\right),
$$

where $C_{1}$ is a constant depending on $T$.

2) Let $x_{0} \in H$ and $k \in L^{2}\left(0, T ; V^{*}\right), T>0$. Then there exists a unique solution $x$ of (LE) belonging to

$$
L^{2}(0, T ; V) \cap W^{1,2}\left(0, T ; V^{*}\right) \subset C([0, T] ; H)
$$

and satisfying

$$
\|x\|_{L^{2}(0, T ; V) \cap W^{1,2}\left(0, T ; V^{*}\right)} \leq C_{1}\left(\left|x_{0}\right|+\|k\|_{L^{2}\left(0, T ; V^{*}\right)}\right),
$$

where $C_{1}$ is a constant depending on $T$.

Lemma 2.3. Suppose that $k \in L^{2}(0, T ; H)$ and $x(t)=\int_{0}^{t} S(t-s) k(s) d s$ for $0 \leq t \leq T$. Then there exists a constant $C_{2}$ such that

$$
\begin{gathered}
\|x\|_{L^{2}(0, T ; D(A))} \leq C_{1}\|k\|_{L^{2}(0, T ; H)}, \\
\|x\|_{L^{2}(0, T ; H)} \leq C_{2} T\|k\|_{L^{2}(0, T ; H)},
\end{gathered}
$$

and

$$
\|x\|_{L^{2}(0, T ; V)} \leq C_{2} \sqrt{T}\|k\|_{L^{2}(0, T ; H)} .
$$

Proof. The assertion (9) is immediately obtained by (7). Since

$$
\begin{aligned}
\|x\|_{L^{2}(0, T ; H)}^{2} & =\int_{0}^{T}\left|\int_{0}^{t} S(t-s) k(s) d s\right|^{2} d t \leq M \int_{0}^{T}\left(\int_{0}^{t}|k(s)| d s\right)^{2} d t \\
& \leq M \int_{0}^{T} t \int_{0}^{t}|k(s)|^{2} d s d t \leq M \frac{T^{2}}{2} \int_{0}^{T}|k(s)|^{2} d s,
\end{aligned}
$$

it follows that

$$
\|x\|_{L^{2}(0, T ; H)} \leq T \sqrt{M / 2}\|k\|_{L^{2}(0, T ; H)} .
$$

From (2), (9), and (10) it holds that

$$
\|x\|_{L^{2}(0, T ; V)} \leq C_{0} \sqrt{C_{1} T}(M / 2)^{1 / 4}\|k\|_{L^{2}(0, T ; H)} .
$$

So, if we take a constant $C_{2}>0$ such that

$$
C_{2}=\max \left\{\sqrt{M / 2}, C_{0} \sqrt{C_{1}}(M / 2)^{1 / 4}\right\},
$$

the proof is complete. 
Consider the following initial value problem for the abstract semilinear parabolic equation

$$
\begin{cases}\frac{d}{d t} x(t) & =A x(t)+\int_{0}^{t} k(t-s) g(s, x(s), u(s)) d s+B u(t), \\ x(0) & =x_{0} .\end{cases}
$$

Let $U$ be some Hilbert space and the controller operator $B$ be a bounded linear operator from $U$ to $H$.

Let $g: \mathbb{R}^{+} \times V \times U \rightarrow H$ be a nonlinear mapping satisfying the following:

(F1) For any $x \in V, u \in U$ the mapping $g(\cdot, x, u)$ is strongly measurable;

(F2) There exist positive constants $L_{0}, L_{1}, L_{2}$ such that

(i) $|g(t, x, u)-g(t, \hat{x}, \hat{u})| \leq L_{1}|| x-\hat{x}\left\|+L_{2}\right\| u-\hat{u} \|_{U}$,

(ii) $|g(t, 0,0)| \leq L_{0}$

for all $t \in \mathbb{R}^{+}, x, \hat{x} \in V$, and $u, \hat{u} \in U$.

For $x \in L^{2}(0, T ; V)$, we set

$$
f(t, x, u)=\int_{0}^{t} k(t-s) g(s, x(s), u(s)) d s,
$$

where $k$ belongs to $L^{2}(0, T)$

Lemma 2.4. Let $x \in L^{2}(0, T ; V)$ for any $T>0$. Then $f(\cdot, x, u) \in L^{2}(0, T ; H)$ and

(12)

$$
\begin{aligned}
& \| f(\cdot, x, u)) \|_{L^{2}(0, T ; H)} \\
\leq & L_{0}\|k\|_{L^{2}(0, T)} T / \sqrt{2}+\|k\|_{L^{2}(0, T)} \sqrt{T}\left(L_{1}\|x\|_{L^{2}(0, T ; V)}+L_{2}\|u\|_{L^{2}(0, T ; U)}\right) .
\end{aligned}
$$

Moreover if $x, \hat{x} \in L^{2}(0, T ; V)$, then

$$
\begin{aligned}
& \|f(\cdot, x, u)-f(\cdot, \hat{x}, \hat{u})\|_{L^{2}(0, T ; H)} \\
\leq & \|k\|_{L^{2}(0, T)} \sqrt{T}\left(L_{1}\|x-\hat{x}\|_{L^{2}(0, T ; V)}+L_{2}\|u-\hat{u}\|_{L^{2}(0, T ; U)}\right) .
\end{aligned}
$$

Proof. From (F1), (F2), and using the Hölder inequality, it is easily seen that

$$
\begin{aligned}
& \|f(\cdot, x, u)\|_{L^{2}(0, T ; H)} \\
\leq & \|f(\cdot, 0,0)\|+\|f(\cdot, x, u)-f(\cdot, 0,0)\| \\
\leq & \left(\int_{0}^{T}\left|\int_{0}^{t} k(t-s) g(s, 0,0) d s\right|^{2} d t\right)^{1 / 2} \\
& +\left(\int_{0}^{T}\left|\int_{0}^{t} k(t-s)\{g(s, x(s), u(s))-g(s, 0,0)\} d s\right|^{2} d t\right)^{1 / 2} \\
\leq & L_{0}\|k\|_{L^{2}(0, T)} T / \sqrt{2}+\|k\|_{L^{2}(0, T)} \sqrt{T}\|g(\cdot, x, u)-g(\cdot, 0,0)\|_{L^{2}(0, T ; H)} \\
\leq & L_{0}\|k\|_{L^{2}(0, T)} T / \sqrt{2}+\|k\|_{L^{2}(0, T)} \sqrt{T}\left(L_{1}\|x\|_{L^{2}(0, T ; V)}+L_{2}\|u\|_{L^{2}(0, T ; U)}\right) .
\end{aligned}
$$

The proof of (13) is similar. 
Theorem 2.5. Under the assumptions (F1), and (F2) for the nonlinear mapping $f$, as given by

$$
f(t, x, u)=\int_{0}^{t} k(t-s) g(s, x(s), u(s)) d s,
$$

there exists a unique solution $x$ of (SE) such that

$$
x \in L^{2}(0, T ; V) \cap W^{1,2}\left(0, T ; V^{*}\right) \subset C([0, T] ; H)
$$

for any $x_{0} \in H$. Moreover, there exists a constant $C_{3}$ such that

$$
\|x\|_{L^{2}(0, T ; V) \cap W^{1,2}\left(0, T ; V^{*}\right)} \leq C_{3}\left(\left|x_{0}\right|+\|u\|_{L^{2}(0, T ; U)}\right) .
$$

Proof. Let us fix $T_{0}>0$ satisfying

$$
C_{2} L_{1} T_{0}\|k\|_{L^{2}(0, T)}<1
$$

with the constant $C_{2}$ in Lemma 2.3. Let $y$ be the solution of

$$
y(t)=S(t) \phi^{0}+\int_{0}^{t} S(t-s)\{f(s, x(s), u(s))+B u(s)\} d s .
$$

We are going to show that $x \mapsto y$ is strictly contractive from $L^{2}\left(0, T_{0} ; V\right)$ to itself. Let $y, \hat{y}$ belong to $V$ with the same initial condition in $\left[0, T_{0}\right]$. Then, noting that $x(s)-\hat{x}(s)=0$ for $s \in\left[0, T_{0}\right]$, from assumption (F1), (11) and

$$
y(t)-\hat{y}(t)=\int_{0}^{t} S(t-s)\{f(s, x(s), u(s))-f(s, \hat{x}(s), u(s))\} d s
$$

we have

$$
\begin{aligned}
\|y-\hat{y}\|_{L^{2}\left(0, T_{0} ; V\right)} & \leq C_{2} \sqrt{T_{0}}\|f(\cdot, x, u)-f(\cdot, \hat{x}, u)\|_{L^{2}\left(0, T_{0} ; H\right)} \\
& \leq C_{2} L_{1} T_{0}\|k\|_{L^{2}\left(0, T_{0}\right)}\|x(\cdot)-\hat{x}(\cdot)\|_{L^{2}\left(0, T_{0} ; V\right)}
\end{aligned}
$$

So by virtue of the condition (15) the contraction mapping principle gives that the solution of (SE) exists uniquely in $\left[0, T_{0}\right]$. Let $x$ be a solution of $(\mathrm{SE})$ and $x_{0} \in H$. Then there exists a constant $C_{1}$ such that

$$
\left\|S(t) x_{0}\right\|_{L^{2}\left(0, T_{0} ; V\right)} \leq C_{1}\left|x_{0}\right|
$$

in view of Proposition 2.2. Let

$$
x_{1}(t)=\int_{0}^{t} S(t-s)\{f(s, x(s), u(s))+B u(s)\} d s .
$$

Then from (11), it follows

$$
\text { (17) } \begin{aligned}
& \left\|x_{1}\right\|_{L^{2}\left(0, T_{0} ; V\right)} \\
\leq & C_{2} \sqrt{T_{0}}\|f(\cdot, x, u)+B u\|_{L^{2}\left(0, T_{0} ; H\right)} \\
\leq & C_{2} \sqrt{T}_{0}\left(L_{1} \sqrt{T_{0}}\|k\|_{L^{2}(0, T)}\|x\|_{L^{2}\left(0, T_{0} ; V\right)}+\|f(\cdot, 0, u)+B u\|_{L^{2}\left(0, T_{0} ; H\right)}\right) .
\end{aligned}
$$


Thus, combining (16) with (17) we have

$$
\begin{aligned}
\|x\|_{\left.L^{2}\left(0, T_{0} ; V\right)\right)} \leq(1 & \left.-C_{2} L_{1} T_{0}\|k\|_{L^{2}(0, T)}\right)^{-1}\left(C_{1}\left|x_{0}\right|\right. \\
& \left.+C_{2} \sqrt{T_{0}}\|f(\cdot, 0, u)+B u\|_{L^{2}\left(0, T_{0} ; H\right)}\right) .
\end{aligned}
$$

Now from

$$
\begin{aligned}
\left|x\left(T_{0}\right)\right|= & \left|S\left(T_{0}\right) \phi^{0}\right|+\left|\int_{0}^{T_{0}} S\left(T_{0}-s\right)\{f(s, x(s), u(s))+B u(s)\} d s\right| \\
\leq & M\left|x_{0}\right|+M L_{1} \sqrt{T_{0}}|| k\left\|_{L^{2}(0, T)}|| x\right\|_{\left.L^{2}\left(0, T_{0} ; V\right)\right)} \\
& +M \sqrt{T_{0}}|| f(\cdot, 0, u)+B u \|_{L^{2}\left(0, T_{0} ; H\right)},
\end{aligned}
$$

since the condition (15) is independent of initial values, the solution of (SE) can be extended to the interval $\left[0, n T_{0}\right]$ for every natural number $n$. An analogous estimate to (14) holds for the solution in $\left[0, n T_{0}\right]$, and hence for the initial value $x_{n T_{0}}$ in the interval $\left[n T_{0},(n+1) T_{0}\right]$.

\section{Approximate controllability of semilinear systems}

Let $x(T ; f, u)$ be a state value of the system (SE) at time $T$ corresponding to the nonlinear term $f$ and the control $u$. We define the reachable sets for the system (SE) as follows:

$$
\begin{aligned}
& R_{T}(f)=\left\{x(T ; f, u): u \in L^{2}(0, T ; U)\right\}, \\
& R_{T}(0)=\left\{x(T ; 0, u): u \in L^{2}(0, T ; U)\right\} .
\end{aligned}
$$

Definition 3.1. The system (SE) is said to be approximately controllable in the time interval $[0, T]$ if for every desired final state $x_{1} \in H$ and $\epsilon>0$ there exists a control function $u \in L^{2}(0, T ; U)$ such that the solution $x(T ; f, u)$ of (SE) satisfies $\left|x(T ; f, u)-x_{1}\right|<\epsilon$, that is, if $\overline{R_{T}(f)}=H$ where $\overline{R_{T}(f)}$ is the closure of $R_{T}(f)$ in $H$, then the system (SE) is called approximately controllable at time $T$.

Let $u \in L^{1}(0, T ; Y)$. Then it is well known that

$$
\lim _{h \rightarrow 0} h^{-1} \int_{0}^{h}\|u(t+s)-u(t)\|_{Y} d s=0
$$

for almost all point of $t \in(0, T)$.

Definition 3.2. The point $t$ which permits (18) to hold is called the Lebesgue point of $u$.

First we consider the approximate controllability of the system (SE) in case where the controller $B$ is the identity operator on $H$ under the Lipschitz conditions (F1), (F2) on the nonlinear operator $f$. So, $H=U$ obviously. Consider 
the linear system given by

$$
\begin{cases}\frac{d}{d t} y(t) & =A y(t)+u(t) \\ y(0) & =x_{0}\end{cases}
$$

and the following semilinear control system

$$
\begin{cases}\frac{d}{d t} x(t) & =A x(t)+f(t, x(t), v(t))+v(t), \\ x(0) & =x_{0} .\end{cases}
$$

Theorem 3.1. Under the assumptions (F1) and (F2) we have

$$
R_{T}(0) \subset \overline{R_{T}(f)} .
$$

Therefore, if the linear system (19) with $f=0$ is approximately controllable, then so is the semilinear system (20).

Proof. Let $y(t)$ be solution of (19) corresponding to a control $u$. First, we show that there exists a $v \in L^{2}(0, T ; H)$ such that

$$
\left\{\begin{array}{l}
v(t)=u(t)-f(t, y(t), v(t)), \quad 0<t \leq T \\
v(0)=u(0)
\end{array}\right.
$$

Let $T_{0}$ be a Lebesgue point of $u, v$ so that

$$
L_{2} \sqrt{T}_{0}\|k\|_{L^{2}\left(0, T_{0}\right)}<1 .
$$

For a given $u \in L^{2}(0, T ; H)$, we define a mapping

$$
W: L^{2}(0, T ; H) \rightarrow L^{2}(0, T ; H)
$$

by

$$
(W v)(t)=u(t)-f(t, y(t), v(t)), \quad 0<t \leq T_{0} .
$$

It follows readily from definition of $W$ that

$$
\begin{aligned}
\left\|W v_{1}-W v_{2}\right\|_{L^{2}\left(0, T_{0} ; H\right)}^{2} & =\left\|f\left(\cdot, y, v_{2}\right)-f\left(\cdot, y, v_{1}\right)\right\|_{L^{2}\left(0, T_{0} ; H\right)}^{2} \\
& \leq L_{2}^{2} T_{0}\|k\|_{L^{2}\left(0, T_{0}\right)}^{2}\left\|v_{2}-v_{1}\right\|_{L^{2}\left(0, T_{0} ; H\right)}^{2}
\end{aligned}
$$

whence

$$
\left\|W v_{1}-W v_{2}\right\|_{L^{2}\left(0, T_{0} ; H\right)} \leq L_{2} \sqrt{T}_{0}|| k\left\|_{L^{2}\left(0, T_{0}\right)}|| v_{2}-v_{1}\right\|_{L^{2}\left(0, T_{0} ; H\right)} .
$$

By a well known the contraction mapping principle $W$ has a unique fixed point $v$ in $L^{2}\left(0, T_{0} ; H\right)$ if the condition $(21)$ is satisfied. Let

$$
v(t)=u(t)-f(t, y(t), v(t)) .
$$


Then from (F1), (F2) and Theorem 2.5, it follows

$$
\begin{aligned}
\|v\|_{L^{2}\left(0, T_{0} ; H\right)} \leq & \|f(\cdot, y, v)+u\|_{L^{2}\left(0, T_{0} ; H\right)} \\
\leq & \sqrt{T}_{0}\|k\|_{L^{2}\left(0, T_{0}\right)}\left(L_{1}\|y\|_{L^{2}\left(0, T_{0} ; V\right)}+L_{2}\|v\|_{L^{2}\left(0, T_{0} ; H\right)}\right) \\
& +\|f(\cdot, 0,0)+u\|_{L^{2}\left(0, T_{0} ; H\right)} \\
\leq & \sqrt{T}_{0}\|k\|_{L^{2}\left(0, T_{0}\right)}\left\{L_{1} C_{1}\left(\left|x_{0}\right|+\|u\|_{L^{2}\left(0, T_{0} ; U\right)}\right)\right. \\
& \left.+L_{2}\|v\|_{L^{2}\left(0, T_{0} ; H\right)}\right\}+\|f(\cdot, 0,0)+u\|_{L^{2}\left(0, T_{0} ; H\right)}
\end{aligned}
$$

Thus, from which we have

$$
\begin{aligned}
\|v\|_{\left.L^{2}\left(0, T_{0} ; H\right)\right) \leq} & \left(1-L_{2}{\sqrt{T_{0}}}\|k\|_{L^{2}\left(0, T_{0}\right)}\right)^{-1}\left\{\sqrt { T } _ { 0 } \| k \| _ { L ^ { 2 } ( 0 , T _ { 0 } ) } L _ { 1 } C _ { 1 } \left(\left|x_{0}\right|\right.\right. \\
& \left.\left.+\|u\|_{L^{2}(0, T ; U)}\right)+\|f(\cdot, 0,0)+u\|_{L^{2}\left(0, T_{0} ; H\right)}\right\} .
\end{aligned}
$$

And we obtain

$$
\begin{aligned}
& \left|v\left(T_{0}\right)\right| \\
= & \left|f\left(T_{0}, y\left(T_{0}\right), v\left(T_{0}\right)\right)-u\left(T_{0}\right)\right| \\
\leq & \mid \int_{0}^{T_{0}} k\left(T_{0}-s\right)\{g(s, y(s), v(s)-g(s, 0,0)\} d s \mid \\
& +\left|\int_{0}^{T_{0}} k\left(T_{0}-s\right) g(s, 0,0) d s+u\left(T_{0}\right)\right| \\
\leq & \|k\|_{L^{2}\left(0, T_{0}\right)}|| g(\cdot, y, v)-g(\cdot, 0,0) \|_{L^{2}\left(0, T_{0} ; H\right)} \\
& +L_{0}|| k \|_{L^{2}\left(0, T_{0}\right)} \sqrt{T}_{0}+\left|u\left(T_{0}\right)\right| \\
\leq & \|k\|_{L^{2}\left(0, T_{0}\right)}\left(L_{1}|| y\left\|_{\left.L^{2}\left(0, T_{0} ; V\right)\right)}+L_{2}\right\| v \|_{L^{2}\left(0, T_{0} ; H\right)}+L_{0} \sqrt{T_{0}}\right)+\left|u\left(T_{0}\right)\right| .
\end{aligned}
$$

If $2 T_{0}$ is a Lebesgue point of $u, v$, then we can solve the equation in $\left[T_{0}, 2 T_{0}\right]$ with the initial value $v\left(T_{0}\right)$ and obtain an analogous estimate to (22) and (23). If not, we can choose $T_{1} \in\left[T_{0}, 2 T_{0}\right]$ to be a Lebesgue point of $u, v$. Since the condition (21) is independent of initial values, the solution can be extended to the interval $\left[T_{1}, T_{1}+T_{0}\right]$, and so we have showed that there exists a $v \in L^{2}(0, T ; H)$ such that $v(t)=u(t)-f(t, y(t), v(t))$. Let $v(t)=u(t)-f(t, y(t), v(t))$ and let $y$ be a solution of (19) corresponding to a control $u$. Consider the following semilinear system

$$
\begin{cases}\frac{d}{d t} x(t) & =A x(t)+f(t, x(t), v(t))+u(t)-f(t, y(t), v(t)), 0<t \leq T \\ x(0) & =x_{0} .\end{cases}
$$

The solution of (19) and (24), respectively, can be written as

$$
y(t)=S(t) x_{0}+\int_{0}^{t} S(t-s) u(s) d s
$$


and

$$
\begin{aligned}
x(t)= & S(t) x_{0}+\int_{0}^{t} S(t-s) u(s) d s \\
& +\int_{0}^{t} S(t-s)\{f(s, x(s), v(s))-f(s, y(s), v(s))\} d s .
\end{aligned}
$$

Then from Theorem 2.5 it is easily seen that $x(\cdot) \in C([0, T] ; H)$, that is, $x(s) \rightarrow$ $x(t)$ as $s \rightarrow t$ in $H$. Let $\epsilon>0$ be given. For $t \geq \epsilon$, set

$$
\begin{aligned}
x^{\epsilon}(t)= & S(t) x_{0}+\int_{0}^{t-\epsilon} S(t-s) u(s) d s \\
& +\int_{0}^{t-\epsilon} S(t-s)\left\{f\left(s, x^{\epsilon}(s), v(s)\right)-f(s, y(s), v(s))\right\} d s .
\end{aligned}
$$

Then we have

$$
\begin{aligned}
x(t)-x^{\epsilon}(t)= & \int_{t-\epsilon}^{t} S(t-s) u(s) d s-\int_{t-\epsilon}^{t} S(t-s) f(s, y(s), v(s)) d s \\
& +\int_{t-\epsilon}^{t} S(t-s) f(s, x(s), v(s)) d s \\
& +\int_{0}^{t-\epsilon} S(t-s)\left\{f(s, x(s), v(s))-f\left(s, x^{\epsilon}(s), v(s)\right)\right\} d s .
\end{aligned}
$$

So, as seen in the proof of Theorem 2.5, for some constant $T_{0}>0$ satisfying $C_{2} L_{1} T_{0}\|k\|_{L^{2}(0, T)}<1$, we see easily that

$$
\begin{aligned}
\left\|x-x^{\epsilon}\right\|_{L^{2}\left(0, T_{0} ; V\right)} \leq & C_{2} \sqrt{\epsilon}\|u\|_{L^{2}\left(0, T_{0} ; H\right)}+C_{2} L_{1} T_{0}\|k\|_{L^{2}(0, T)}\left\|x-x^{\epsilon}\right\|_{L^{2}\left(0, T_{0} ; V\right)} \\
& +C_{2} \sqrt{\epsilon} L_{1} \sqrt{T}_{0}\|k\|_{L^{2}(0, T)}\|x-y\|_{L^{2}\left(0, T_{0} ; V\right)} .
\end{aligned}
$$

By the step by step method, we know that $x^{\epsilon} \rightarrow x$ as $\epsilon \rightarrow 0$ in $L^{2}(0, T ; V)$ $(T>0)$ for $\epsilon<t<T$. From (6) it follows that

$$
\begin{aligned}
& \left\|x^{\epsilon}-y\right\|_{L^{2}(0, T ; V)}^{2} \\
= & \int_{0}^{T}\left|\int_{0}^{t+s-\epsilon} S(t+s-\tau)\left\{f\left(\tau, x^{\epsilon}(\tau), v(\tau)\right)-f(\tau, y(\tau), v(\tau))\right\} d \tau\right|^{2} d s \\
\leq & \left(M L_{1}\|k\|_{L^{2}(0, \tau)}\right)^{2} \int_{0}^{T}\left(\int_{0}^{t+s-\epsilon}(t+s-\tau)^{-1 / 2}\left\|x^{\epsilon}-y\right\|_{L^{2}(0, \tau ; V)} d \tau\right)^{2} d s \\
\leq & \left(M L_{1}\|k\|_{L^{2}(0, \tau)}\right)^{2} \int_{0}^{T} \log \left(\frac{t}{\epsilon}\right)\left\|x^{\epsilon}-y\right\|_{L^{2}(0, \tau ; V)}^{2} d \tau d s \\
\leq & T\left(M L_{1}\|k\|_{L^{2}(0, \tau)}\right)^{2} \log \left(\frac{t}{\epsilon}\right) \int_{0}^{T}\left\|x^{\epsilon}-y\right\|_{L^{2}(0, \tau ; V)}^{2} d \tau .
\end{aligned}
$$

By using Gronwall's inequality, independently of $\epsilon$, we get $x^{\epsilon}=y$ in $L^{2}(0, T ; V)$ for almost all $\epsilon \leq t \leq T$, and $x^{\epsilon}(t)=y(t)$ in $H$. Therefore, noting that 
$x(\cdot), y(\cdot) \in C([0, T ; H])$, every solution of the linear system with control $u$ is also a solution of the semilinear system with control $w$, that is, we have that $R_{T}(0) \subset \overline{R_{T}(f)}$.

From now on, we consider the initial value problem for the semilinear parabolic equation (SE). Let $U$ be some Hilbert space and the controller operator $B$ be a bounded linear operator from $U$ to $H$.

Theorem 3.2. Let us assume that there exists a constant $\beta>0$ such that $\|B u\| \geq \beta\|u\|$ for all $u \in L^{2}(0, T ; U)$, and the assumptions (F1), (F2), and $R(f) \subset R(B)$ be satisfied. Then we have

$$
R_{T}(0) \subset \overline{R_{T}(f)} .
$$

Proof. Consider the linear system given by

$$
\begin{cases}\frac{d}{d t} y(t) & =A y(t)+u(t) \\ y(0) & =x_{0}\end{cases}
$$

and the following semilinear control system

$$
\begin{cases}\frac{d}{d t} x(t) & =A x(t)+f(t, x(t), v(t))+B v(t), \\ x(0) & =x_{0} .\end{cases}
$$

Let $y$ be a solution of (25) corresponding to a control $u$. Set $v(t)=u(t)-$ $B^{-1} f(t, y(t), v(t))$. Then as seen in Theorem 3.1, we know that $v \in L^{2}(0, T ; U)$. Consider the following semilinear system

$$
\begin{cases}\frac{d}{d t} x(t) & =A x(t)+f(t, x(t), v(t))+B u(t)-f(t, y(t), v(t)), 0<t \leq T \\ x(0) & =x_{0} .\end{cases}
$$

If we define $x^{\epsilon}, y$ as in proof of Theorem 3.1, then we get

$$
x^{\epsilon}(t)-y(t)=\int_{0}^{t-\epsilon} S(t-s)\left\{f\left(s, x^{\epsilon}, v(s)\right)-f(s, y, v(s))\right\} d s .
$$

So using Gronwall's inequality, as in Theorem 3.1, we obtain that $R_{T}(0) \subset$ $\overline{R_{T}(f)}$.

Example. We consider the semilinear heat equation dealt with by Zhou [9], and Naito [4]. Let

$$
\begin{aligned}
& H=L^{2}(0, \pi), V=H_{0}^{1}(0, \pi), V^{*}=H^{-1}(0, \pi), \\
& a(u, v)=\int_{0}^{\pi} \frac{d u(x)}{d x} \frac{\overline{d v(x)}}{d x} d x
\end{aligned}
$$

and

$$
A=d^{2} / d x^{2} \quad \text { with } \quad D(A)=\left\{y \in H^{2}(0, \pi): y(0)=y(\pi)=0\right\} .
$$


We consider the following retarded functional differential equation

$$
\begin{cases}\frac{d}{d t} y(x, t) & =A y(x, t)+\int_{0}^{t} k(t-s) g(s, x(s), u(s)) d s+B u(t), \\ y(t, 0) & =y(t, \pi)=0, \quad t>0, \\ y(0, x) & =\phi^{0}(x), \quad y(x, s)=\phi^{1}(x, s), \quad-h \leq s<0,\end{cases}
$$

where $k$ belongs to $L^{2}(0, T)$. The eigenvalue and the eigenfunction of $A$ are $\lambda_{n}=-n^{2}$ and $\phi_{n}(x)=\sin n x$, respectively. Let

$$
\begin{aligned}
& U=\left\{\sum_{n=2}^{\infty} u_{n} \phi_{n}: \sum_{n=2}^{\infty} u_{n}^{2}<\infty\right\}, \\
& B u=2 u_{2} \phi_{1}+\sum_{n=2}^{\infty} u_{n} \phi_{n} \text { for } \quad u=\sum_{n=2}^{\infty} u_{n} \in U .
\end{aligned}
$$

It is easily seen that the operator $B$ is one to one and $\mathrm{R}(B)$ is closed. It follows that the operator $B$ satisfies hypothesis as in Theorem 3.2. For example, consider the nonlinear term $f$ given by

$$
g(t, y, u)=\alpha(t)\left(\left\|D_{x} y\right\| \phi_{1}(x)+\|u\| \phi_{2}(x)\right), \quad \alpha(t) \in C([0, T]) .
$$

Then $f$ is not uniformly bounded and $\mathrm{R}(g) \subset \mathrm{R}(B)$ and from Theorem 3.2 it follows that the system of (SE1) is approximately controllable. In case where $B=I$ we obtain the approximate controllability of (SE1) without restrictions such as the uniform boundedness and inequality constraints for Lipschitz constant of $f$ or compactness of $S(t)$.

\section{References}

[1] G. Di Blasio, K. Kunisch, and E. Sinestrari, $L^{2}$-regularity for parabolic partial integrodifferential equations with delay in the highest-order derivatives, J. Math. Anal. Appl. 102 (1984), no. 1, 38-57.

[2] J. P. Dauer and N. I. Mahmudov, Approximate controllability of semilinear functional equations in Hilbert spaces, J. Math. Anal. Appl. 273 (2002), no. 2, 310-327.

[3] J. M. Jeong, Y. C. Kwun, and J. Y. Park, Approximate controllability for semilinear retarded functional-differential equations, J. Dynam. Control Systems 5 (1999), no. 3, 329-346.

[4] K. Naito, Controllability of semilinear control systems dominated by the linear part, SIAM J. Control Optim. 25 (1987), no. 3, 715-722.

[5] N. Sukavanam and N. K. Tomar, Approximate controllability of semilinear delay control systems, Nonlinear Funct. Anal. Appl. 12 (2007), no. 1, 53-59.

[6] H. Tanabe, Equations of Evolution, Translated from the Japanese by N. Mugibayashi and H. Haneda. Monographs and Studies in Mathematics, 6. Pitman (Advanced Publishing Program), Boston, Mass.-London, 1979.

[7] H. Triebel, Interpolation Theory, Function Spaces, Differential Operators, NorthHolland, 1978.

[8] M. Yamamoto and J. Y. Park, Controllability for parabolic equations with uniformly bounded nonlinear terms, J. Optim. Theory Appl. 66 (1990), no. 3, 515-532.

[9] H. X. Zhou, Approximate controllability for a class of semilinear abstract equations, SIAM J. Control Optim. 21 (1983), no. 4, 551-565. 
Jin-MUN JEONG

Division of Mathematical Sciences

Pukyong National University

Pusan 608-737, Korea

E-mail address: jmjeong@pknu.ac.kr

Han-Geul Kim

Division of Mathematical Sciences

Pukyong National University

PusAn 608-737, Korea

E-mail address: gry99@naver.com 